${ }^{\ominus} \mathrm{B}$. Johnson, T. Kuga, and H. M. Gladney, IBM J. Res. Develop. 13, 36 (1969).

${ }^{10} \mathrm{H}$. J. Gerritsen and E. S. Sabisky, Phys. Rev. 125, 1853 (1962).

${ }_{11}$ T. C. Ensign, T.-T. Chang, and A. H. Kahn Phys. Rev. 188, 703 (1969).

${ }^{12}$ R. E. Dietz, H. Kamimura, M. D. Sturge, and A. Yariv, Phys. Rev. 132, 1559 (1963).

${ }^{13}$ Z. Sroubek and K. Zdansky, J. Chem. Phys. 44, 3078 (1966). ${ }^{14} \mathrm{I}$. Siegel and J. A. Lorenc, J. Chem. Phys, 45, 2315 (1966).

${ }^{15}$ M. de Wit and A. R. Reinberg, Phys. Rev. 163, 261 (1967).

${ }^{16}$ R. E. Coffman, J. Chem. Phys. 48, 609 (1968).

${ }^{17}$ K. Zdansky, Phys. Rev. 177, 490 (1969).

18 J. R. Pilbrow and J. M. Spaeth, Phys. Status Solidi 20, 225 (1967).

${ }^{19}$ A. H. Maki and B. R. McGarvey, J. Chem. Phys. 29, 31 (1958).

${ }_{20}$ D. Kivelson and R. Neiman, J. Chem. Phys. 35, 149 (1961).

${ }^{21}$ H. R. Gersmann and J. D. Swalen, J. Chem. Phys. 36, 3221 (1962).

${ }_{22}$ H. A. Kuska and M. T. Rogers, J. Chem. Phys. 43, 1744 (1965); 40, $910(1964)$

${ }^{23}$ G. F. Kokoszka, H. C. Allen, and G. Gordon, J. Chem. Phys. 42,3730 (1965).

${ }^{24}$ T. R. Reddy and R. Stinivasan, J. Chem. Phys. 43, 1404 (1965).

${ }_{25}$ G. R. Wagner, R. T. Schumacher, and S. A. Friedberg, Phys. Rev. 150, 226 (1966).

${ }_{26}$ R. H. Dunhill, J. R. Pilbrow, and T. D. Smith, J. Chem. Phys. 45, 1474 (1966).

${ }_{27}$ G. F. Kokoszka, H. C. Allen, and G. Gordon, J. Chem. Phys. 46, 3020 (1967).

${ }^{28}$ T. Chiang, J. Chem. Phys. 48, 1814 (1968).

${ }^{29} \mathrm{M}$. Abkowitz, I. Chen, and J. H. Sharp, J. Chem. Phys. 48, 4561 (1968).
${ }^{30}$ G. Rist, J. Ammeter, and H. H. Gunthard, J. Chem. Phys. 49,2210 (1968).

${ }^{31}$ A. Abragam and M. H. L. Pryce, Proc. Roy. Soc. (London) A206, 164 (1951).

${ }^{32}$ A. Abragam and M. H. L. Pryce, Proc. Roy. Soc. (London) A205, 135 (1951).

${ }^{33}$ R. E. Watson and A. J. Freeman, in Hyperfine Interactions, A. J. Freeman and R. B. Frankel, Eds. (Academic Press Inc., New York, 1967), Chap. 2.

${ }^{34}$ J. W. Stout and S. A. Reed, J. Am. Chem. Soc. 76, 5279 (1954).

${ }^{35}$ W. H. Baur, Acta Cryst. 11, 488 (1958).

${ }^{36}$ C. Billy and H. M. Haendler, J. Am. Chem. Soc. 79, 1049 (1957).

${ }^{37}$ References $10,11,13,18$, and 26 cover crystal systems with the $d_{z^{2}}$ orbital as the ground state. Note that we have oriented our $x$ and $y$ axis at $45^{\circ}$ from the common convention with $D_{4 h}$.

${ }^{38}$ W. Hayes and J. Wilkins, Proc. Roy. Soc. (London) A281, 340 (1964)

${ }^{39} \mathrm{P}$. Bagus (private communication).

${ }^{40} \mathrm{~J}$. P. Goldsborough and T. R. Koehler, Phys. Rev, 133, A135 (1964).

${ }^{41}$ L. C. Allen, S. H. Glarum, H. M. Gladney, J. Chem. Phys. 40, 3135 (1964).

${ }^{42} \mathrm{~J}$. S. Griffith The Theory of Transition Metal Ions (Cambridge Universiy Press, New York, 1964).

${ }^{43}$ See, for example Refs. 19-21.

${ }^{44} \mathrm{~W}$. Marshall, Proceedings of the First International Conference. on Paramagnetic Rseoonance, Jerusalem, 1962, W. Low, Ed (Academic Press Inc., New York, 1963), Vol. 1, p. 347.

${ }_{45}$ H. M. Gladney and A. Veillard, Phys. Rev. 180, 385 (1969).

${ }^{46} \mathrm{R}$. N. Kortzeborn (private communication)

${ }^{47}$ J. Owen and J. H. M. Thornley, Rept. Progr. Phys. 29, 675 (1966).

THE JOURNAL OF CHEMICAL PHYSICS

VOLU M E 52 , N UM B ER 8

15 A P R I L $19 ; 0$

\title{
Behavior of Solutions of Linear Macromolecules in Steady Shear Flow with Superposed Oscillations
}

\author{
D. Colin Evans, * Harold R. Warner, Jr., Willitam R. Ramakka, $\dagger$ and R. Byron Bird \\ Chemical Engineering Department and Rheology Research Center, University of Wisconsin, Madison, Wisconsin 53706
}

(Received 8 December 1969)

\begin{abstract}
The rotational diffusion equation has been solved for a suspension of rigid dumbbells in a velocity field which is the superposition of steady shearing flow (large deformation) and an infinitesimal oscillatory motion (very small deformation). An expression is obtained for the associated shear stresses and the results are expressed in terms of a complex viscosity, appropriately modified for the superposed steady shearing flow. The rigid dumbbell model gives a maximum in the real part of the complex viscosity as well as negative values for the imaginary part at low frequencies. Such behavior has been observed experimentally. The elastic dumbbell model (two beads connected by a Hookean spring) does not give results which are qualitatively similar to the experimental data.
\end{abstract}

\section{INTRODUCTION}

Of current interest in the mechanical response of macromolecular fluids is the behavior in a flow field $\tau_{x}(y, t)=\kappa(t) y$, which is a superposition of a steady shearing flow $\left(v_{x}=\kappa_{m} y\right)$ and an infinitesimal oscillatory motion

$$
v_{x}=\operatorname{Re}\left\{\kappa^{0} e^{i \omega t}\right\} y .
$$

Here $\kappa_{m}$ is a real constant, and $\kappa^{0}$ is a complex quantity with $\left|\kappa^{0}\right|$ quite small; $\omega$ is the frequency of the superimposed oscillatory motion.

Experimental data for polymer solutions have been reported by Osaki, Tamura, Kurata, and Kotaka, ${ }^{1}$ by
Booij, ${ }^{2}$ and by Macdonald. ${ }^{3}$ Osaki et al. used polystyrene in toluene, polymethylmethacrylate in diethylphthalate, and poly- $n$-butylmethacrylate in diethylphthalate. Booij's data were for aluminum dilaurate in decalin and a rubbery ethylene-propylene copolymer in decalin. Macdonald's experiments were performed on several polystyrenes in several Aroclors and on polyisobutylene in Primol. Shortly after the original publication of the Japanese group, Macdonald and Bird $^{4}$ showed that a nonlinear viscoelastic constitutive equation was capable of describing quantitatively their data on both the non-Newtonian viscosity and the superposed flow material functions. Markovitz ${ }^{5}$ has also 
examined this flow from a more general continuum mechanics viewpoint.

It is the purpose of this communication to show how the superposed flow problem can be solved for a suspension of rigid dumbbells using the dilute polymer solution theory including Brownian motion but neglecting hydrodynamic interaction (see Ref. 6 for the bibliographical listing of the previous publications by Kirkwood, Prager, Giesekus, Kotaka, and others).

\section{EQUATIONS FOR THE RIGID DUMBBELL KINETIC THEORY}

For unsteady-state shearing flows with $v_{x}=\kappa(t) y$ the "rotational diffusion equation" for the orientational distribution function $\psi(\theta, \phi, t)$ for rigid dumbbells is ${ }^{6}$

$$
\begin{aligned}
6 \lambda \frac{\partial \psi}{\partial t} & =\frac{1}{S} \frac{\partial}{\partial \theta}\left(S \frac{\partial \psi}{\partial \theta}\right)+\frac{1}{S^{2}} \frac{\partial^{2} \psi}{\partial \phi^{2}} \\
& \quad-6 \lambda \kappa\left[\frac{s c}{S} \frac{\partial}{\partial \theta}\left(S^{2} C \psi\right)-\frac{\partial}{\partial \phi}\left(s^{2} \psi\right)\right] \\
& \equiv \Lambda \psi-6 \lambda_{\kappa}(t) \Omega \psi,
\end{aligned}
$$

in which $\Lambda$ and $\Omega$ are linear operators defined by the identity above, and $S=\sin \theta, C=\cos \theta, s=\sin \phi$, and $c=\cos \phi$. The time constant $\lambda=\zeta L^{2} / 12 k T$ contains $\zeta$, the friction constant for one bead of the dumbbell, and $L$ the length of the dumbbell.

Once $\psi(\theta, \phi, t)$ has been obtained from Eq. (1), the shear stress can be calculated from ${ }^{6}$

$$
\tau_{y x}-\tau_{y x, 8}=\frac{n_{0} \zeta L^{2}}{4 J}\left[\frac{\partial}{\partial t}\left\langle S^{2} s c\right\rangle-\kappa(t)\left\langle S^{2} s^{2}\right\rangle\right],
$$

where $\langle Q\rangle=\iint Q \psi S d \theta d \phi$ and $J=\iint \psi S d \theta d \phi$; furthermore $\tau_{y x, s}=-\eta_{s} \kappa(t)$ where $\eta_{s}$ is the solvent viscosity, and $n_{0}$ is the number density of the dumbbells in the suspension. form

For the superposed flow considered here $\kappa(t)$ has the

$$
\kappa(t)=\kappa_{m}+\operatorname{Re}\left\{\kappa^{0} e^{i \omega t}\right\},
$$

where $\kappa_{m}$ is the mean value of the velocity gradient about which small oscillations are performed. The shear stress response will be of the form

$$
\tau_{y x}-\tau_{y x, 8}=\tau_{m}+\operatorname{Re}\left\{\tau^{0} e^{i \omega t}\right\}+\cdots
$$

We will consider only the sinusoidal response and not concern ourselves with higher harmonics. The quantities $\tau_{m}$ and $\tau^{0}$ are

$$
\begin{aligned}
\tau_{m} & =-\left(\eta-\eta_{s}\right) \kappa_{m}, \\
\tau^{0} & =-\left(\eta_{\kappa}^{*}-\eta_{s}\right) \kappa^{0} .
\end{aligned}
$$

Equation (5) states that the mean shear stress is related to the mean velocity gradient in the usual way, with $\eta$ being the non-Newtonian viscosity [Ref. 6, Eq. (6)]

$$
\begin{aligned}
\eta-\eta_{s}=n_{0} k T \lambda\left[1-(18 / 35)\left(\lambda \kappa_{m}\right)^{2}\right. \\
\left.+(1326 / 1925)\left(\lambda \kappa_{m}\right)^{4}-\cdots\right]
\end{aligned}
$$

which is valid up to $\lambda \kappa_{m}=0.47$ within $1 \%$ of the numerical solution of Kirkwood and Plock. ${ }^{7}$ Equation (6) which relates the complex amplitudes $\tau^{0}$ and $\kappa^{0}$ is a defining equation for $\eta_{x}{ }^{*}$ the "complex viscosity for superposed steady shear flow," as defined by Osaki, Tamura, Kurata, and Kotaka. ${ }^{1}$ It is customary to decompose this quantity into real and imaginary parts $\eta_{x}{ }^{*}=\eta_{k}{ }^{\prime}-i \eta_{k}{ }^{\prime \prime}$. When $\kappa_{m}=0$, then $\eta_{k}^{*}, \eta_{k}^{\prime}$, and $\eta_{k}{ }^{\prime \prime}$ become the same as the quantities $\eta^{*}, \eta^{\prime}$, and $\eta^{\prime \prime}$ in small amplitude oscillatory motion. ${ }^{8}$

\section{SOLUTION OF THE ROTATIONAL DIFFUSION EQUATION}

We wish now to find a solution to Eq. (1) with $\kappa(t)$ given by Eq. (3). The form of the solution will be

$$
\psi(\theta, \phi, t)=\sum_{k=0}^{\infty}\left(6 \lambda \kappa_{m}\right)^{k}\left[\psi_{k}+\operatorname{Re}\left\{6 \kappa^{0} \lambda \phi_{k} e^{i \omega t}\right\}\right] .
$$

Here the $\psi_{k}(\theta, \phi)$ are the functions which arise in the solution of Eq. (1) for steady shearing flow with $\kappa(t)=$ $\kappa_{m}$; for this case the distribution function has the form $\psi=\psi_{0}+\left(6 \lambda_{\kappa_{m}}\right) \psi_{1}+\left(6 \lambda \kappa_{m}\right)^{2} \psi_{2}+\cdots$ and the $\psi_{k}$ have to satisfy the differential equations $\Lambda \psi_{k}=\Omega \psi_{k-1}$. The first few $\psi_{k}$ are

$$
\begin{aligned}
& \psi_{0}=1, \\
& \psi_{1}=\frac{1}{4} S^{2} S_{2}, \\
& \psi_{2}=\frac{1}{64} S^{4}+\frac{1}{24} S^{2} c_{2}-\frac{1}{64} S^{4} c_{4},
\end{aligned}
$$

where

$$
s_{k}=\sin k \phi, \quad c_{k}=\cos k \phi .
$$

The $\psi(\theta, \phi)$ and $\psi_{k}$ for steady shear flow as given here differ slightly from those in a previous publication; however, the normalized distribution function $\psi(\theta, \phi) / \iint \psi(\theta, \phi) S d \theta d \phi$ is the same.

When the trial function of Eq. (8) is inserted into Eq. (1), one gets the following equations for the first few $\phi_{k}$ :

$$
\begin{aligned}
& 6 i \lambda \omega \phi_{0}=\Lambda \phi_{0}-\Omega 1, \\
& 6 i \lambda \omega \phi_{1}=\Lambda \phi_{1}-\Omega\left(\psi_{1}+\phi_{0}\right), \\
& 6 i \lambda \omega \phi_{2}=\Lambda \phi_{2}-\Omega\left(\psi_{2}+\phi_{1}\right) .
\end{aligned}
$$

These equations may be solved to give

$$
\begin{aligned}
& \phi_{0}=A_{22}{ }^{0} S^{2} s_{2}, \\
& \phi_{1}=\left(A_{00}{ }^{1}+A_{20}{ }^{1} S^{2}+A_{40}{ }^{1} S^{4}\right)+A_{22}{ }^{1} S^{2} c_{2}+A_{44}{ }^{1} S^{4} c_{4}, \\
& \phi_{2}=\left(A_{22}{ }^{2} S^{2}+A_{42}{ }^{2} S^{4}+A_{62}{ }^{2} S^{6}\right) s_{2}+A_{44}{ }^{2} S^{4} s_{4}+A_{66}{ }^{2} S^{6} s_{6}
\end{aligned}
$$

and the coefficients $A_{p q}$ are given in Table I.

\section{THE SHEAR STRESS AND THE MODIFIED COMPLEX VISCOSITY}

When the expressions in Eqs. (9)-(11) for the $\psi_{k}$ and Eqs. (15)-(17) for the $\phi_{k}$ are substituted into Eq. (8) and the latter inserted into Eq. (2), it is 
found that

$\tau_{y x}-\tau_{y x, 8}=-\frac{3}{2}\left(n_{0} k T \lambda / J\right)\left[\kappa_{m}+\operatorname{Re}\left\{\kappa^{0} e^{i \omega t}\right\}\right] \iint\left[1+\left(6 \lambda \kappa_{m}\right)^{2} \psi_{2}+\left(6 \lambda \kappa_{m}\right) \operatorname{Re}\left\{6 \lambda \kappa^{0} \phi_{1} e^{i \omega t}\right\}\right] S^{3}\left(1-c_{2}\right) d \theta d \phi$

with

$$
+\frac{3}{2}\left(n_{0} k T \lambda / J\right)(\partial / \partial t) \iint \operatorname{Re}\left\{6 \lambda \kappa^{0} e^{i \omega t}\left[\phi_{0}+\left(6 \lambda \kappa_{m}\right)^{2} \phi_{2}\right]\right\} S^{3} s_{2} d \theta d \phi,
$$

$$
J=\iint\left[1+\left(6 \lambda_{\kappa_{m}}\right)^{2} \psi_{2}+\left(6 \lambda \kappa_{m}\right) \operatorname{Re}\left\{6 \lambda \kappa^{0} \phi_{1} e^{i \omega t}\right\}\right] S d \theta d \phi .
$$

After integration and considerable laborious rearrangement one finally obtains for the quantity defined in Eq. (6)

$$
\eta_{*}^{*}-\eta_{s}=n_{0} k T \lambda\left[1-\frac{3}{5} \frac{j}{1+j}+\frac{6}{175}\left(\lambda \kappa_{m}\right)^{2}\left(\frac{-450-465 j-93 j^{2}+49 j^{3}+12 j^{4}}{(1+j)^{3}(10+3 j)}\right)\right],
$$

in which $j=i \lambda \omega$; from this expression one obtains

$$
\begin{gathered}
\eta_{\star}^{\prime}-\eta_{s}=n_{0} k T \lambda\left[\frac{5+2(\lambda \omega)^{2}}{5\left[1+(\lambda \omega)^{2}\right]}+\frac{6}{175}\left(\lambda \kappa_{m}\right)^{2}\left(\frac{-4500+3135(\lambda \omega)^{2}+2361(\lambda \omega)^{4}+742(\lambda \omega)^{6}+36(\lambda \omega)^{8}}{\left(1+(\lambda \omega)^{2}\right)^{3}\left(100+9(\lambda \omega)^{2}\right)}\right)\right], \\
\eta_{k}{ }^{\prime \prime}=n_{0} k T \lambda\left[\frac{3 \lambda \omega}{5\left(1+(\lambda \omega)^{2}\right)}-\frac{6}{175}\left(\lambda_{\kappa_{m}}\right)^{2}\left(\frac{\left.10200(\lambda \omega)+6026(\lambda \omega)^{3}+1887(\lambda \omega)^{5}+81(\lambda \omega)^{7}\right)}{\left(1+(\lambda \omega)^{2}\right)^{3}\left(100+9(\lambda \omega)^{2}\right)}\right)\right] .
\end{gathered}
$$

Some sample curves based on these equations are shown in Figs. 1 and 2.

\section{CONCLUDING COMMENTS}

It will be noted that the curves of $\eta_{k}{ }^{\prime}-\eta_{s}$ calculated to the order shown in Eq. (21) exhibit maxima, although they are not very pronounced. The experimental data of Macdonald ${ }^{3}$ seem to display maxima of this type. At low $\omega$ the calculated $\eta_{k}{ }^{\prime}-\eta_{s}$ values decrease with increasing $\kappa_{m}$, but at high $\omega$ the reverse is true. The data of Macdonald agree with these results at low frequency, but his data do not extend far enough into the high $\omega$ range to make a comparison there.

It is further noted that the function $\eta_{x}{ }^{\prime \prime}$ calculated to the order shown in Eq. (22) has negative values for $\kappa_{m}$ above some critical value, and for low frequencies, just as was predicted by the earlier calculations of Macdonald and Bird. ${ }^{4}$ Negative values were not reported by Osaki et al., ${ }^{1}$ but the subsequent measurements of Booij ${ }^{2}$ and of Macdonald ${ }^{3}$ did reveal such negative values. In connection with this and the preceding paragraph it must be mentioned that terms of order $\kappa_{m}^{4}$ and higher have been omitted.

In the limit as $\kappa_{m}$ goes to zero, the expressions in Eqs. (20)-(22) simplify properly to the formulas obtained previously for small amplitude oscillatory motion. It should be noted that the rigid dumbbell model

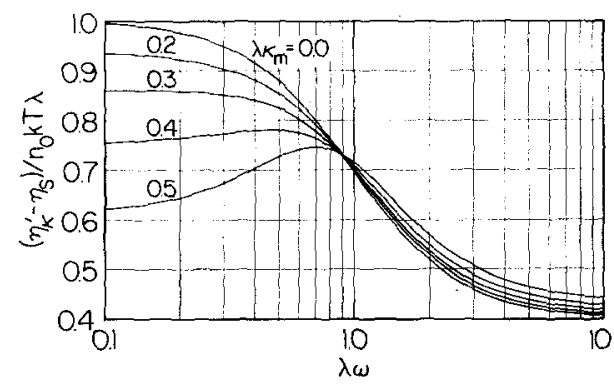

FIG. 1. Behavior of $\eta_{r^{\prime}}$ as computed from Eq. (21). indicates that $\eta^{\prime}(\omega)-\eta s$ goes to a nonzero limit as the frequency becomes infinite. Recent experimental data $a^{9,10}$ have also shown this kind of behavior.

In the limit of zero frequency, the expression for the shear stress must simplify (for the special case of $\kappa^{0}$ real) to the steady state flow result with velocity gradient $\kappa_{m}+\kappa_{0}$; from Eq. (7) this should be

$$
\begin{aligned}
\lim _{\omega \rightarrow 0} \tau_{\nu x}= & -\eta_{s}\left(\kappa_{m}+\kappa^{0}\right) \\
- & {\left[1-(18 / 35) \lambda^{2}\left(\kappa_{m}+\kappa^{0}\right)^{2}+\cdots\right]\left(\kappa_{m}+\kappa^{0}\right) } \\
= & -\eta_{s}\left(\kappa_{m}+\kappa^{0}\right)-\left[\left(\kappa_{m}+\kappa^{0}\right)\right. \\
& \left.-(18 / 35) \lambda^{2} \kappa_{m}^{3}-(54 / 35) \lambda^{2} \kappa_{m}^{2} \kappa^{0}+\cdots\right],
\end{aligned}
$$

if we discard terms $O\left(\kappa^{02}\right)$ and higher. From Eqs. (4) -(6) and Eq. (21) we get

$$
\begin{aligned}
\lim _{\omega \rightarrow 0} \tau_{y x}= & -\eta_{8}\left(\kappa_{m}+\kappa^{0}\right) \\
& -n_{0} k T \lambda\left[1-(18 / 35)\left(\lambda \kappa_{m}\right)^{2}+\cdots\right] \kappa_{m} \\
& \quad-n_{0} k T \lambda\left[1-(54 / 35)\left(\lambda \kappa_{m}\right)^{2}+\cdots\right] \kappa^{0}
\end{aligned}
$$

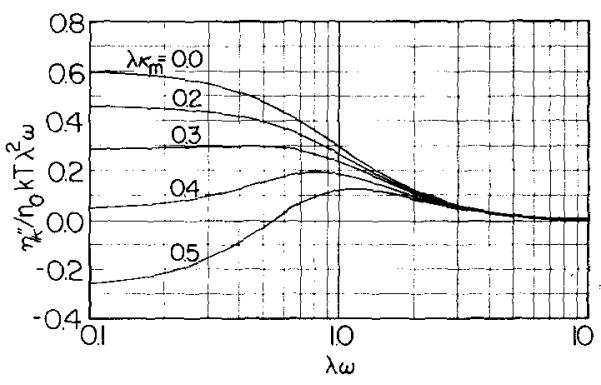

FIG, 2. Behavior of $\eta_{\kappa}^{\prime \prime}$ as computed from Eq. (22). 
TABLE I. Coefficients in Eqs. (15)-(17) [for brevity $j \equiv i \lambda \omega]$.

$$
\begin{aligned}
& A_{22^{0}}=\frac{1}{4} \frac{1}{(1+j)} \quad A_{20}=-\frac{1}{80} \frac{(2+j) j}{(1+j)^{2}\left(1+\frac{3}{10} j\right)} \\
& A_{00}{ }^{1}=-\frac{1}{120} \frac{(2+j)}{(1+j)^{2}\left(1+\frac{3}{10} j\right)} \quad A_{22}{ }^{1}=\frac{1}{24} \frac{(2+j)}{(1+j)^{2}} \\
& A_{40}{ }^{1}=-A_{44}{ }^{1}=\frac{1}{64} \frac{(2+j)}{(1+j)\left(1+\frac{3}{10} j\right)} \\
& A_{66}{ }^{2}=-\frac{1}{1536} \frac{(1+j)\left(1+\frac{3}{10} j\right)+(2+j)}{(1+j)\left(1+\frac{1}{7} j\right)\left(1+\frac{3}{10} j\right)}=-\frac{1}{3} A_{62}{ }^{2} \\
& A_{44^{2}}=\frac{1}{3840} \frac{16(1+j)^{2}\left(1+\frac{3}{10} j\right)+(2+j)(16+9 j)}{(1+j)^{2}\left(1+\frac{3}{10} j^{2}\right)} \\
& A_{42}{ }^{2}=\frac{1}{640} \frac{2(1+j)\left[(1+j)\left(1+\frac{3}{10} j\right)+(2+j)\right]-(2+j)(2+3 j)\left(1+\frac{1}{7} j\right)}{(1+j)^{2}\left(1+\frac{3}{10} j\right)^{2}\left(1+\frac{1}{7} j\right)}-\frac{1}{320}\left(1+\frac{3}{10} j\right)^{-1} \\
& A_{22}{ }^{2}=\frac{1}{6}(1+j)^{-1}\left[12 A_{42}{ }^{2}-\frac{1}{24}-\frac{13}{240} \frac{(2+j)}{(1+j)^{2}\left(1+\frac{3}{10} j\right)}\right]
\end{aligned}
$$

[where the second term on the right side is from Eq. (7) and the last from Eq. (21)]. To $O\left(\kappa^{0}\right)$ this is in agreement with Eq. (23).

In conclusion it should be pointed out that the Lodge elastic liquid ${ }^{11}$ model, obtained from a network theory, gives $\eta_{\kappa}{ }^{*}$ exactly equal to $\eta^{*}$ for all $\kappa_{m}$, and is hence not in agreement with the experimental observations. Lodge and $\mathrm{Wu}^{12}$ have very recently shown that the beadspring models (with Hookean springs) of the type used by Rouse and Zimm surprisingly lead to a constitutive equation which has the same form as that of a Lodge elastic liquid. Therefore an elastic dumbbell with a Hookean spring would give results inconsistent with experimental data, whereas the rigid dumbbell displays qualitatively the correct behavior. The inadequacy of the bead-spring models has been recently pointed out in connection with elongational flow ${ }^{13}$ and light scattering. ${ }^{14}$

\section{ACKNOWLEDGMENTS}

The authors wish to acknowledge the financial support received from National Science Foundation Grant GK-1275 and Petroleum Research Fund Grant 1758-C of the American Chemical Society. One of the authors (H. R. W., Jr.) wishes to thank the National Science
Foundation for a graduate fellowship during the course of this work.

* Present address: Department of Applied Mathematics, University of Liverpool, Liverpool, England.

$\dagger$ Present address: ESSO Research and Engineering Co., Florham Park, N.J.

${ }^{1}$ K. Osaki, M. Tamura, M. Kurata, and T. Kotaka, J. Phys. Chem. 69, 4183 (1965).

${ }^{2}$ H. C. Booij, Rheol. Acta 5, 215, 222 (1966).

${ }^{3}$ I. F. Macdonald, Ph.D. thesis, University of Wisconsin, 1968.

${ }^{4}$ I. F. Macdonald and R. B. Bird, J. Phys. Chem. 70, 2068 (1966).

${ }^{5}$ H. Markovitz, Proc. Intern. Cong. Rheol. 5th, Kyoto, Japan, October 1968 (unpublished)

${ }^{6}$ R. B. Bird, H. R. Warner, Jr., and W. R. Ramakka, J. Chem. Phys. 52, 2001 (1970).

${ }^{7} \mathrm{~J}$. G. Kirkwood and R. J. Plock, J. Chem. Phys. 24, 665 (1966) ; there are errors in this paper as pointed out by E. Paul, J. Chem. Phys. 51, 1271 (1969)

${ }^{8} \mathrm{~J}$. D. Ferry, Viscoelastic Properties of Polymers (John Wiley \& Sons, Inc., New York, 1970), 2nd ed.

${ }^{9} \mathrm{~J}$. D. Ferry, L. A. Holmes, J. Lamb, and A. J. Matheson, J. Phys. Chem. 70, 1685 (1966).

${ }^{10}$ R. S. Moore, H. J. McSkimin, C. Gieniewski, and P. Andreatch, Jr., J. Chem. Phys. 50, 5088 (1969).

${ }^{11}$ A. S. Lodge, Elastic Liquids (Ácademic Press Inc., New York, 1964), pp. 113-114.

${ }^{12} \mathrm{~A}$. S. Lodge and Y. Wu, University of Wisconsin Mathematics Research Center Technical Summary Rept. No. 1040, 28 January 1970.

${ }^{13}$ R. B. Bird, M. W. Johnson, Jr., and C. F. Curtiss, J. Chem. Phys. 51, 3023 (1969).

${ }_{14}$ F. R. Cottrell, E. W. Merrill, and K. A. Smith, J. Polymer Sci. A2 7, 1415 (1969). 